https://helda.helsinki.fi

\title{
Oral lichen sclerosus : a systematic review of reported cases and two new cases
}

Kakko, Tuomas

2018-05

Kakko , T , Salo , T \& Siponen , M K 2018 , ' Oral lichen sclerosus : a systematic review of reported cases and two new cases ' , International Journal of Dermatology , vol. 57 , no. 5 , pp. 521-528 . https://doi.org/10.1111/ijd.13870

http://hdl.handle.net/10138/301262

https://doi.org/10.1111/ijd.13870

unspecified

publishedVersion

Downloaded from Helda, University of Helsinki institutional repository.

This is an electronic reprint of the original article.

This reprint may differ from the original in pagination and typographic detail.

Please cite the original version. 


\title{
Oral lichen sclerosus: a systematic review of reported cases and two new cases
}

\author{
Tuomas Kakko ${ }^{1}$, BDS, Tuula Salo ${ }^{2,3,4}$, DDS, PhD, and Maria K. Siponen ${ }^{2,5,6}$, DDS, PhD
}

${ }^{1}$ Institute of Dentistry, Faculty of Medicine, University of Oulu, Oulu, Finland, ${ }^{2}$ Cancer and Translational Medicine Research Unit, Faculty of Medicine, University of Oulu and Oulu University Hospital, Oulu, Finland, ${ }^{3}$ Department of Oral and Maxillofacial Diseases, University of Helsinki, Oulu, Finland, ${ }^{4}$ Medical Research Center, Oulu University Hospital, Oulu, Finland,

${ }^{5}$ Department of Oral and Maxillofacial Diseases, Kuopio University Hospital, Kuopio, Finland, and ${ }^{6}$ Institute of Dentistry, Faculty of Health Sciences, University of Eastern Finland, Kuopio, Finland

\author{
Correspondence \\ Maria K. Siponen, DDS, PHD \\ Institute of Dentistry \\ University of Eastern Finland \\ Faculty of Health Sciences \\ P.O Box 1627 \\ 70211 Kuopio \\ Finland \\ E-mails: marsip@uef.fi; \\ maria.siponen@kuh.fi
}

Conflict of interest: None to declare.

\begin{abstract}
Lichen sclerosus (LS) is a chronic inflammatory mucocutaneous disease with uncertain etiology. It occurs as white plaque-like lesions mostly in the anogenital skin. Oral mucosal involvement is extremely rare. This study aims to summarize the features of published oral lichen sclerosus (OLS) and two new cases. A systematic search of the English literature from 1955 to 2016 was performed in MEDLINE, Scopus, and Web of Science, and crossreferences were searched manually. Search phrases included "lichen sclerosus," "mouth," "oral," "lip," "palate," "floor of mouth," "tongue," "gingiva," "buccal mucosa," and "mouth diseases." Cases with clinical and histopathological confirmation of diagnosis of OLS were included. A total of 41 (39 published and 2 new) histologically confirmed OLS cases were available. The median age of OLS patients was 31 years, and $66 \%$ of the patients were female. Most of the OLS lesions were asymptomatic. They were located in the labial mucosa $(n=20)$, lip $(n=15)$, buccal mucosa $(n=14)$, gingiva $(n=12)$, tongue $(n=12)$, and palate $(n=7)$. OLS is rare and typically presents as asymptomatic, white, plaque-like lesions. Malignant transformation of preexisting OLS has not been reported.
\end{abstract}

\section{Introduction}

Lichen sclerosus (LS) is a relatively rare chronic inflammatory mucocutaneous disease of uncertain etiology. The prevalence of LS is estimated to be $0.1-0.3 \%{ }^{1}$ The disease is more common in women and may be found in both children and adults with a typical onset before puberty in children, after menopause in women, and in the fourth decade in men. ${ }^{2}$ LS was first described in 1887 by Hallopeau in his article "Du lichen plan et particulièrement de sa forme atrophique: lichen plan scléreux." ${ }^{3}$ LS involving the oral mucosa was first described by Miller in $1957 .{ }^{4}$ LS has been previously called lichen sclerosus et atrophicus and balanitis xerotica obliterans, but the name was changed to lichen sclerosus in 1976 by the International Society for the Study of Vulvar Disease, because atrophy is not always present in the lesions. ${ }^{5}$

LS presents clinically as ivory or porcelain-white, polygonal, flat-topped papules, plaques, or atrophic patches. ${ }^{6}$ LS occurs mainly in the anogenital area but may arise anywhere on the skin with upper back, chest, and breasts affected most frequently. ${ }^{6}$ About $60 \%$ of women with genital LS have symptoms. ${ }^{6}$ The symptoms of genital LS are itching, fissuring, erosion, dysuria, urethral and vaginal discharge, dyspareunia, and burning pain in females and hemorrhage, shallow erosions, phimosis, paraphimosis, and urethral meatus stenosis in men. Cutaneous LS is usually asymptomatic but can feature pain and pruritus. Genital LS is associated with an increased risk of malignant transformation. ${ }^{6}$ Differently, malignant change in extragenital LS lesions is extremely rare. ${ }^{7}$

The etiological factors of LS are still uncertain, but infections (e.g. Borrelia burgdorferi and Epstein-Barr virus infections), autoimmune reactions, irritation (urine), hormones, and genetic factors have been suggested to play a role in the pathogenesis. ${ }^{2}$ A growing consensus is that LS has an autoimmune etiology in genetically susceptible individuals. ${ }^{2,8}$

The most characteristic histopathological feature of LS is hyalinization of the lamina propria. ${ }^{8}$ Early LS lesions show 


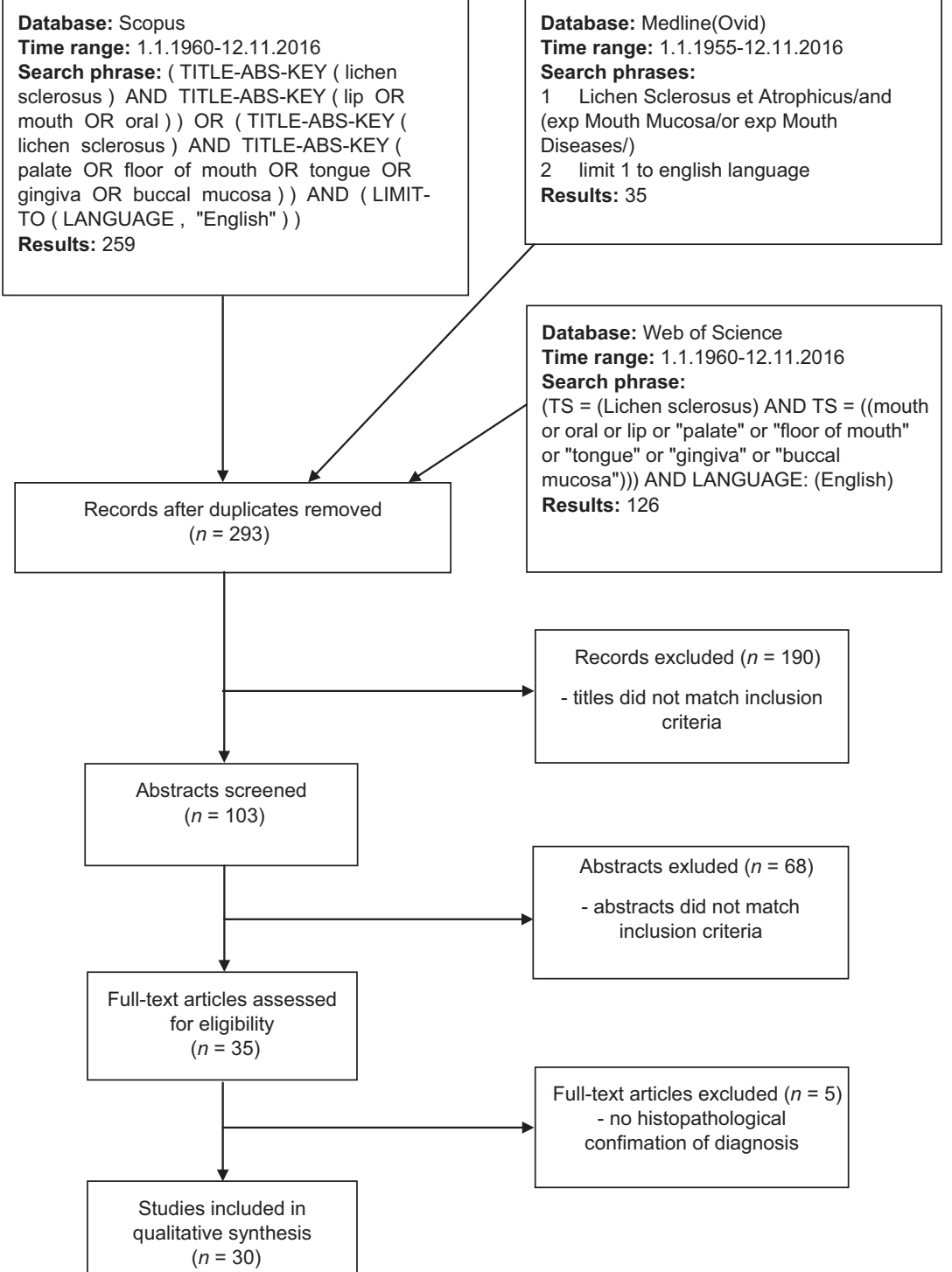

\begin{tabular}{|l|l|} 
Records after duplicates removed \\
$(n=293)$
\end{tabular}

Database: Medline(Ovid)

Time range: 1.1.1955-12.11.2016

Search phrases:

1 Lichen Sclerosus et Atrophicus/and

(exp Mouth Mucosa/or exp Mouth

Diseases/)

2 limit 1 to english language

Results: 35

Fig. 1 Flowchart of database searching

subepidermal edema with a band-like lymphocytic infiltrate and vacuolar alteration of keratinocytes. ${ }^{6,8}$ More advanced lesions also show epidermal hyperkeratosis and atrophy with effacement of the rete ridges, and loss of elastic fibers. ${ }^{2,6,8}$ Clefting and blisters may result from subepidermal edema and interface dermatitis. $^{8}$

Oral LS is very rare. To the authors' knowledge, there are only 39 histologically proven reported cases of OLS in the English literature. However, LS affecting the lip may be underreported. $^{9}$ OLS is rarely symptomatic but can cause pain, soreness, pruritus, and tightness when opening the mouth. ${ }^{10-17}$ Gingival lesions may cause gingival recession, loss of periodontal attachment, and even tooth mobility. ${ }^{13,14,16}$ Malignant transformation of OLS has not been reported.

The aim of this study was to summarize the demographic and clinicopathologic data of LS involving the oral cavity and lips for the first time in a systematic review. In addition, we report two new cases of OLS.

\section{Materials and methods}

The review was done according to the PRISMA statement guidelines. Three databases were searched for English case reports and case series of OLS (Fig. 1). Search phrases included "lichen sclerosus," "mouth," "oral," "lip," "palate," "floor of mouth," "tongue," "gingiva," "buccal mucosa," and "mouth diseases." The inclusion criteria were case reports or case series with clinical and histopathological description diagnostic of OLS. Exclusion criteria were LS of skin or anogenital mucosa without oral lesions or no histological confirmation of clinical diagnosis. After duplicates were removed, the publications were reviewed by TK and MS. 

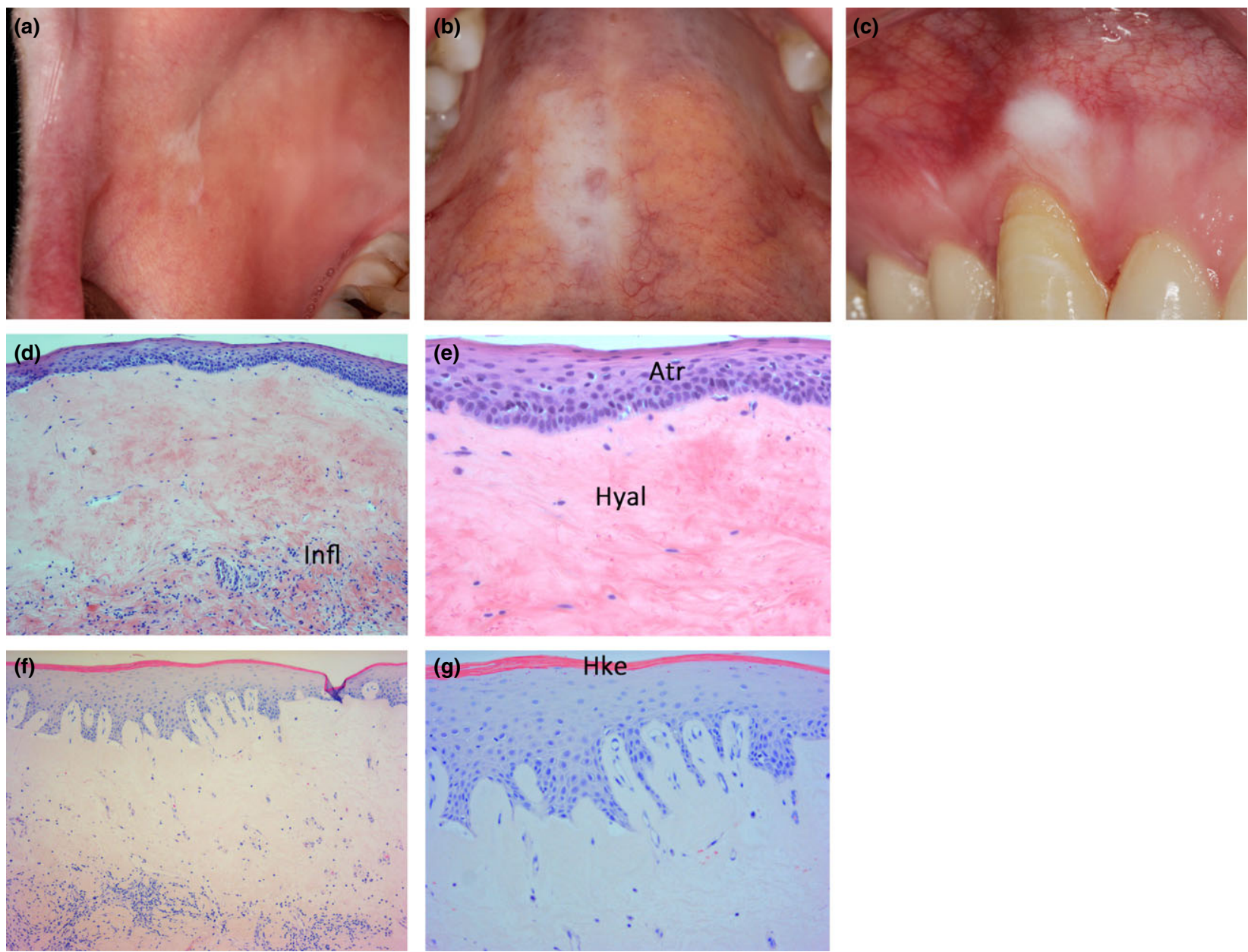

Fig. 2 Clinical and histopathological features of OLS.

Case 1: Whitish plaque-like lesions in the buccal mucosa (a), palate (b) and gingiva (c). Atrophic (Atr) epithelium and hyalinized connective tissue (Hyal) with underlying lymphocytic inflammatory infiltrate (Infl) $(d, e)$

Case 2: Hyperkeratotic epithelium (Hke), slight hydropic degeneration of the basal cells, subepithelial homogenization, and lymphocytic inflammatory infiltrate in the connective tissue $(f, g)$. H\&E, original magnification $\times 100(d, f), \times 200(e, g)$

Clinical information of the present two cases were collected from the patient records of Kuopio University Hospital, Finland (Case 1), and from information included in a consultation case from Kainuu Central Hospital, Finland (Case 2).

Histopathological slides of the cases were retrieved and reexamined. The data were analyzed with descriptive statistical methods.

The study plan was approved by the Regional Ethics Committee of the Northern Ostrobothnia Hospital District. The systematic review was registered to the PROSPERO register (registration number CRD42017064920).

\section{Case reports}

\section{Case 1}

A 28-year-old female was referred to the Department of Oral and Maxillofacial Diseases at Kuopio University Hospital owing to oral mucosal changes. The patient had multiple sclerosis (MS), allergies to pollen and sulfonamide, and was a smoker.

The patient had a history of pain, edema, and vesiculation in the right side of the hard palate. A biopsy had been taken 14 months previously from the symptomatic area. The histopathological diagnoses were nonspecific chronic inflammation and chronic lichenoid inflammation with a suspicion of pemphigoid.

Clinical examination revealed diffuse, plaque-like, white lesions on the right buccal mucosa near the corner of the mouth, on the right side of the palate, and on the buccal gingiva of the upper right canine (Fig. $2 \mathrm{a}-\mathrm{C}$ ). The lesions were not pruritic nor painful. The patient did not have lesions in the genital area or skin.

The palatal lesion was biopsied, and histological examination showed atrophic stratified squamous epithelium with a thin parakeratinized layer and occasionally visible stratum 
granulosum. Hydropic degeneration of the basal cells and slight clefting in the epithelium-connective tissue junction were seen in areas. There was scantiness of cells and hyalinization in the lamina propria. A dense inflammatory infiltrate was present in deeper connective tissue. The histological features were consistent with LS. Differential diagnosis included morphea. Immunofluorescence (IF) examination of fresh tissue from the palate was also performed, and it showed linear $\lg G$, $\operatorname{lgM}$, and fibrin deposits along the basal membrane. Indirect IF did not reveal circulating anti-skin (intraepidermal or basement membrane) antibodies. No treatment was administered because of the patient being pregnant.

After 13 months, at follow-up examination the previous oral lesions were unchanged, and a new small, white, plaque-like lesion was noticed on the left side of the tongue (not shown). All the lesions were asymptomatic. A new biopsy was performed from the gingival lesion in the upper right canine region. Histopathological examination showed a thin parakeratinized layer over thinned stratified squamous epithelium, basal destructive changes, clefting along the basal membrane, hyalinization of connective tissue, and chronic inflammatory cell infiltrate in deeper areas of the connective tissue (Fig. 2d, e). IF examination of the second biopsy was negative for antibody deposits. The histopathological diagnosis was LS.

\section{Case 2}

A 51-year-old generally healthy, nonsmoking woman with two oral lesions was seen at Kainuu Central Hospital in Kajaani. One of the lesions was bullous and located on the palatal mucosa, whereas the other lesion, resembling leukoplakia, was found on maxillary alveolar mucosa. The clinical features were suspicious for mucous membrane pemphigoid.

The palatal lesion was excised. Histological examination showed subepithelial blister formation and homogenization, hyalinization of collagen fibers, hydropic degeneration of the basal cells, band-like lymphocytic inflammatory infiltrate in the connective tissue, telangiectasia, and lack of elastic fibers (Fig. 2f, g). The amount of melanocytes was within normal range. The patient was given the diagnosis of LS. Later, LS lesions were also found in the vulva and perineum. At a followup examination after 18 months, no new lesions were found.

\section{Results}

Literature review yielded a total of 39 histologically proven cases of OLS (Fig. 1). Most papers reported only one case $(n=25)$, one reported six cases, ${ }^{17}$ and four reported two cases. ${ }^{18-21}$ The present report adds two new cases of histologically confirmed OLS, raising the total number so far to 41 .

Summary of the demographic and clinical characteristics of the OLS cases is presented in Table 1. More detailed data about the patient characteristics are shown in Table S1. OLS was reported from four continents (Asia, Europe, North
Table 1 Summary of clinical characteristics of OLS patients.

\begin{tabular}{lc}
\hline Characteristic & $\begin{array}{c}\text { Literature review } \\
(\boldsymbol{n}=\mathbf{3 9})+\text { present } \\
\text { cases }(\boldsymbol{n}=\mathbf{2})\end{array}$ \\
\hline Mean age/Median age & $34 / 31$ (range $7-70)$ \\
Sex F/M (\%) & $27 / 14(66 / 34 \%)$ \\
Smoker & \\
$\quad$ yes & $5(12 \%)$ \\
no & $21(51 \%)$ \\
not specified & $15(37 \%)$ \\
Lesion site & \\
labial mucosa & $20(49 \%)$ \\
lip & $15(37 \%)$ \\
buccal mucosa & $14(34 \%)$ \\
gingiva & $12(29 \%)$ \\
tongue & $12(29 \%)$ \\
palate & $7(17 \%)$ \\
Symptoms, if reported yes/no (\%) & $12 / 26(32 \% / 68 \%)$ \\
Genital lesions, if reported & $7(18 \%)$ \\
Skin lesions, if reported & $12(31 \%)$ \\
Treatment, if reported yes/no (\%) & $25 / 13(66 \% / 34 \%)$ \\
topical corticosteroids & $10(26 \%)$ \\
intralesional corticosteroid injections & $6(16 \%)$ \\
topical tacrolimus/pimecrolimus & $5(13 \%)$ \\
surgery & $5(13 \%)$ \\
systemic corticosteroids & $2(5 \%)$ \\
other medication ${ }^{\mathrm{a}}$ & $10(26 \%)$ \\
\hline &
\end{tabular}

aOther medication included colchicine $(n=2)$, Solcoseryl, griseofulvin, topical testosterone propionate, benzydamine hydrochloride, topical antibiotics, emollients, methotrexate, pantothenyl alcohol, topical vitamin A and systemic vitamins A \& D.

America, and South America). Mean age among the patients was 34 years (range 7-70). Sixty-six percent of the patients were female. Five of the patients, including present case 1, were smokers, 21 were nonsmokers, and for the rest, the reports did not specify whether or not the patients smoked cigarettes. Twenty-one (70\%) patients were generally healthy. Nine (30\%) patients were reported to have medical problems: asthma ( $n=3)$, hyperthyroidism $(n=2)$, partial albinism, carcinoma of the cervix, seroresistant syphilis, and dermatitis venenata $(n=1)$, hypertension $(n=1)$, alcoholism $(n=1)$, slight anemia $(n=1)$, and multiple sclerosis $(n=1)$. General medical status was not reported for 11 patients. Only three cases were reported to have allergies. Of note, the vast majority of case reports did not specifically state whether or not the patients had allergies.

Most oral lesions presented as white, homogenous plaques on the oral mucosa or lip. Occasionally, associated telangiectasias $^{20,22,23}$ or erosions ${ }^{11}$ were reported. Labial mucosa, buccal mucosa, and lip were the most common locations of the OLS lesions, followed by gingiva and tongue. Notably, floor of the mouth mucosa was not affected by OLS in any of the patients. The majority of the patients were asymptomatic; only less than one-third had symptoms including pain, pruritus, and tightness when opening the mouth. Gingival involvement by OLS caused 
soreness associated with tooth brushing in some patients. Gingival recessions, periodontal attachment loss, and tooth mobility were also reported. ${ }^{12-14,16}$ In one case, this led to loss of teeth in the affected area. ${ }^{13}$ Thirty-one percent of the patients had skin lesions, and $18 \%$ had genital lesions. The oral LS lesions occurred concurrently with the extraoral lesions in eight cases, after the onset of extraoral lesions in five cases, and before the onset of extraoral lesions in two cases.

Two-thirds of the patients received some form of treatment. The most common medications to manage the OLS symptoms were topical corticosteroids $(n=10)$, intralesional corticosteroids $(n=6)$, and topical calcineurin inhibitors tacrolimus $(n=4)$ and pimecrolimus $(n=1)$. Five patients were treated surgically (Table 1). The other, less commonly used treatments included systemic corticosteroids $(n=2)$, systemic colchicine $(n=2)$, methotrexate, topical testosterone, systemic vitamin D2 with systemic and topical pantothenyl alcohol, Solcoseryl, and griseofulvin. Topical corticosteroids resulted in partial response in $67 \%(6 / 9)$ of the patients, and in no response in $33 \%(3 / 9)$ of the patients (Table S1). In one patient, the response to topical corticosteroid was not reported. Intralesional corticosteroids showed partial response in $67 \%$ (4/6) of the patients, and complete response and no response in one patient each. The patient of Macleod et al. ${ }^{11}$ was treated with griseofulvin after topical corticosteroids failed to provide a response, and it caused some improvement. Systemic corticosteroids used with intralesional corticosteroids yielded a favorable response in one report. Tacrolimus ointment resulted in complete resolution of lesions in one case and partial improvement in one. For two patients, the response for topical tacrolimus was not reported. The use of pimecrolimus (after no response to topical corticosteroid) showed resolution of lesions with remaining atrophy in one report. Surgical excision of the lesion resulted in complete response in $80 \%(4 / 5)$, while in one patient the lesion recurred after excision. For $71 \%$ of the patients, the follow-up duration was available, and the average time of follow-up reported was 24 months (ranging from 0 to 90 months). None of the OLS cases were reported to undergo malignant transformation.

Histopathological and immunofluorescence features of OLS lesions are presented in Table S2. The most consistent histopathological finding was homogenization/hyalinization of collagen in the lamina propria, which was found in all the biopsies. A band-like, patchy or diffuse chronic inflammatory infiltrate was present in the connective tissue, often below the subepithelial hyalinized layer, in all except one case. Epithelial atrophy and basal hydropic degeneration or vacuolization of basal cells were found in 63 and $61 \%$ of the cases, respectively. Sparseness of elastic fibers was reported in $46 \%$ of the biopsies. Hyperkeratosis was present in $41 \%$ of the cases. Other less common histopathological findings were dilated blood vessels $(27 \%)$, subepithelial edema $(10 \%)$, and subepithelial clefts (10\%). Direct IF study was done in four patients (in 5 biopsies) and was negative except in the first biopsy of the
Table 2 Main clinical and histopathological differential diagnoses of OLS.

\begin{tabular}{|c|c|}
\hline Differential diagnosis & Distinguishing features \\
\hline \multicolumn{2}{|l|}{ Clinical } \\
\hline $\begin{array}{l}\text { Chronic hyperplastic } \\
\text { candidiasis }\end{array}$ & $\begin{array}{l}\text { Typically in anterior buccal mucosa } \\
\text { bilaterally, diffuse margins }\end{array}$ \\
\hline Frictional keratosis & $\begin{array}{l}\text { Typically in edentulous alveolar ridge, } \\
\text { diffuse margins }\end{array}$ \\
\hline Leukoplakia & $\begin{array}{l}\text { Wrinkled surface, typically in a middle- } \\
\text { aged or older person, or smoker }\end{array}$ \\
\hline $\begin{array}{l}\text { Lichen planus (plaque } \\
\text { type) }\end{array}$ & $\begin{array}{l}\text { Symmetrical distribution, includes reticular } \\
\text { areas or white striae, and often } \\
\text { erythematous areas, most are } \\
\text { symptomatic }\end{array}$ \\
\hline Morphea & $\begin{array}{l}\text { Extremely rare. Most cases in the anterior } \\
\text { oral cavity with involvement of the lip, } \\
\text { gingiva and alveolar bone. Often } \\
\text { represents linear morphea. }\end{array}$ \\
\hline Oral submucous fibrosis & $\begin{array}{l}\text { Diffuse lesions, stiffness of the oral } \\
\text { mucosa, stomatopyrosis, trismus }\end{array}$ \\
\hline Snuff-dippers pouch & $\begin{array}{l}\text { Wrinkled or fissured surface in the area } \\
\text { where smokeless tobacco is held }\end{array}$ \\
\hline \multicolumn{2}{|l|}{ Histopathological } \\
\hline Morphea & $\begin{array}{l}\text { No focal basal cell degeneration or loss } \\
\text { of elastic fibers in the hyalinized tissue. } \\
\text { Obliteration or narrowing of blood } \\
\text { vessels }\end{array}$ \\
\hline $\begin{array}{l}\text { Lichenoid lesion/lichen } \\
\text { planus }\end{array}$ & $\begin{array}{l}\text { No subepithelial hyalinization. Band-like } \\
\text { lymphocytic infiltrate immediately } \\
\text { subjacent to the epithelium }\end{array}$ \\
\hline
\end{tabular}

present case 1, which showed linear $\lg G$ and $\lg M$ along the basement membrane.

\section{Discussion}

OLS typically presents with whitish, ivory- or porcelain-white macules or plaques with well-demarcated borders. ${ }^{21,24}$ These clinical features may mimic other chronic, white, plaque-type lesions, such as plaque-like lichen planus, leukoplakia, chronic hyperplastic candidiasis, oral submucous fibrosis, and localized scleroderma (morphea). ${ }^{6,12}$ In addition, some OLS cases may show characteristics of autoimmune bullous disease. ${ }^{2}$ Early LS lesions may histologically have overlapping features with lichen planus or psoriasis, ${ }^{2}$ but more mature lesions often show lack of elastic fibers without band of lymphocytes at epithelium-connective tissue junction, unlike lichen planus. ${ }^{12}$ For histological distinction from morphea, Verhoeff's histochemical staining may be used to show the loss of elastic fibers in LS biopsies. The main clinical and histopathological differential diagnoses of OLS are presented in Table 2.

The age range in OLS is wide, with a mean age of 34 . Hence, OLS patients are typically younger than patients with lichen planus, leukoplakia, or chronic hyperplastic candidiasis. OLS patients are often older than oral submucosis fibrosis 
patients, of whom $85 \%$ are under 35 years old, ${ }^{25}$ and child-type morphea patients, whose mean age is $9 .^{26}$ Adult-type morphea patients have, in contrast, a higher mean age (44 years) than OLS patients. ${ }^{26}$

Associated autoimmune diseases were found in a few OLS patients. The present case 1 had MS, which is considered an immune-mediated, possibly autoimmune disease. Two patients had hyperthyroidism, which is typically an autoimmune disease. Of note, many reports did not state whether or not the patients had any comorbidities. A few papers mentioned that the patients had no family history of LS. The family history of possible autoimmune conditions in the patients of this review was not reported.

Controversy still exists about the possible autoimmune nature of LS. ${ }^{27}$ However, female LS patients have an increased prevalence of associated autoimmune diseases, whereas in men, this association is weaker or even nonexistent. ${ }^{28-32}$ The most common associated autoimmune pathosis in LS seems to be thyroid gland disease. Alopecia areata, vitiligo, pernicious anemia, rheumatoid arthritis, localized scleroderma, ulcerative colitis, and psoriasis have less often been associated with LS. ${ }^{2,28}$ Of note, circulating extracellular matrix protein 1 (ECM1) antibodies have been observed more often in LS patients compared to controls. $^{33,34}$

There is some evidence of genetic background for the development of LS. Sherman et al. ${ }^{35}$ found that $12 \%$ of female LS patients had a positive family history of LS. In addition, several major histocompatibility complex genes occur more often in LS patients than in controls. ${ }^{31,32,35-40}$

IF examination of biopsy was performed in four cases, ${ }^{12,14,41}$ and only the present case 1 first showed positive findings: linear IgG, IgM, and fibrin deposits at the basement membrane suggesting pemphigoid. However, the second IF examination 13 months later yielded negative results. The IF findings from the first biopsy of the present case 1 suggest that autoantibodies against a basement membrane antigen may be present in LS. In general, direct IF examination in LS is rare. However, Dickie et $a l .{ }^{42}$ found that a proportion of vulvar LS patients had positive IgM, C3, and fibrin along the basement membrane in direct IF. In addition, circulating antibodies against the basement membrane have been observed in vulvar LS. ${ }^{43,44}$ In this review, the four cases of OLS analyzed did not show circulating autoantibodies (Case 1). ${ }^{13,16,45}$ During follow-up of case 1, no clinical or histopathological features of pemphigoid have been observed. Also, the present case 2 was clinically suspected to have pemphigoid owing to a bullous lesion in the palate. None of the cases included in the literature review mentioned pemphigoid suspicion. However, bullous LS is a known morphological variant of LS, with epidermal atrophy and weakening of the basal cell layer, basement membrane zone, and papillary dermis as a possible mechanism behind the bullae formation. ${ }^{46}$ Also, vacuolar degeneration of the basal cell layer, cleft formation along the basement membrane, and subepithelial edema that have been reported in $\mathrm{OLS}^{23,47}$ may cause clinical bullae.
It is therefore possible that the present cases were bullous forms of LS in their early presentation.

As a result of the small number of patients, randomized controlled trials are difficult to conduct in OLS. Therefore evidencebased treatment recommendations for OLS are not available. Topical corticosteroids are considered to be the first-line symptomatic management of LS. ${ }^{2}$ Recent guidelines on anogenital LS in women recommend potent to very potent topical steroids as a treatment of choice. ${ }^{48}$ In the present review, 16 cases $(42 \%)$ were treated with topical or intralesional corticosteroids, and the results were often favorable. Based on the present review, intralesional corticosteroid treatment may be more efficient than topical treatment. Although the numbers for treated patients were small, surgery and topical calcineurin inhibitors could potentially be of use in the management of OLS. However, it is recommended that only LS patients with a malignancy or scarring process should be treated surgically. ${ }^{2}$ Systemic retinoids have been successfully used in the treatment of vulvar LS. $^{2}$ The two OLS cases treated with oral vitamin A did not show any response. ${ }^{18}$

The risk of genital LS lesions developing malignant change has been estimated to be $0.3-4.9 \%$ for women and $9.3 \%$ for men. ${ }^{2}$ Questioning the relatively high malignant transformation rate of LS, a study by van der Nieuwenhof et al. ${ }^{49}$ shows that a significant proportion of cases initially diagnosed as vulvar LS, and that progressed to squamous cell carcinoma, were on revision in fact diagnosed as vulvar intraepithelial neoplasia. Thus, LS may not be a precancerous condition as such but a chronic scarring process in an environment where carcinogenesis could be enhanced. ${ }^{8}$ No malignant transformation was reported amongst the 41 cases of OLS in this review. However, the follow-up of the patients was generally short.

Among the strengths of this study is the systematic search of three comprehensive databases. There have been other reviews of OLS, ${ }^{17,21,24,50,51}$ but these were not systematic reviews. The weakness of this review is that some relevant articles may have been excluded owing to limiting the language to English, and that there might exist articles not included in the three aforementioned databases. We were able to retrieve information from all the case reports and case series that fulfilled the inclusion criteria; therefore we consider this review to be of low risk of bias regarding data collection. In addition, all the data were analyzed by two researchers.

\section{Conclusions}

OLS is very rare but should be included in the differential diagnosis of white plaque-like lesions of the oral mucosa. In case of symptomatic lesions, topical or intralesional corticosteroids are the first-line therapy. Surgical excision, if feasible, may achieve complete response. Topical calcineurin inhibitors may be used in cases not responding to topical steroids. Malignant potential of OLS remains currently unsolved. 


\section{Acknowledgments}

The assistance of information specialist Sirpa Grekula with the literature search is gratefully acknowledged.

\section{Questions (answers provided following references)}

1. What is the prevalence of oral LS?
a $0.01-0.03 \%$
b $0.1-0.3 \%$
c $0.3-1 \%$
d Unknown

2. The pathogenesis of oral LS is associated with Borrelia burgdorferi -infections.

\section{a True \\ b False}

3. The majority of patients with oral LS also have skin lesions.
a True
b False

4. Which of the following statements are correct?

a Most patients with genital LS present symptoms

b Most patients with oral LS are asymptomatic

c Oral LS may cause tooth loss

d Oral LS is a risk factor for oral cancer

5. Clinical differential diagnosis of oral LS includes:
a erythroplakia
b white sponge nevus
c lichen planus
d leukoplakia

6. Biopsy of oral LS is mandatory for diagnosis.
a True
b False

7. Immunofluorescence examination of the biopsy is recommended in oral LS.

a True

8. The most distinctive histopathological feature of LS is
a epidermal atrophy
b lichenoid interface dermatitis
c subepidermal edema
d superficial dermal collagen homogenization

9. Histopathological differential diagnoses of LS includes:

a bullous pemphigoid

b morphea

c lichen planus

d lupus erythematosus

10. In oral LS, topical calcineurin inhibitors are the treatment of choice.
a True
b False

\section{References}

1 Wallace HJ. Lichen sclerosus et atrophicus. Trans St Johns Hosp Dermatol Soc 1971; 57: 9-30.

2 Fistarol SK, Itin PH. Diagnosis and treatment of lichen sclerosus: an update. Am J Clin Dermatol 2013; 14: 27-47.

3 Hallopeau $\mathrm{H}$. Du lichen plan et particulierement de sa forme atrophique. Ann Dermatol Syph 1887; 8: 790-791.

4 Miller RF. Lichen sclerosus et atrophicus with oral involvement: histopathologic study and dermabrasive treatment. Arch Dermatol 1957; 76: 43-55.

5 The International Society for the Study of Vulvar. Disease. Obstet Gynecol 1976; 47: 122-124.

6 James W, Berger T, Elston D. Andrews' Diseases of the Skin, 11th edn. US: Saunders, 2011.

7 Sergeant A, Vernall N, Mackintosh LJ, et al. Squamous cell carcinoma arising in extragenital lichen sclerosus. Clin Exp Dermatol 2009; 34: e278-e279.

8 Bolognia J, Jorizzo J, Schaffer J. Dermatology, 3rd edn. Philadelphia: Elsevier, 2012.

9 Attili VR, Attili SK. Lichen sclerosus of lips: a clinical and histopathologic study of 27 cases. Int J Dermatol2010; 49: 520-525.

$10 \mathrm{Siar} \mathrm{CH}, \mathrm{Ng} \mathrm{KH}$. Oral lichen sclerosus et atrophicus: report of a case. J Oral Med 1985; 40: 148-150.

11 Macleod RI, Soames JV. Lichen scleroses et atrophicus of the oral mucosa. Br J Oral Maxillofac Surg 1991; 29: 64-65.

12 Buajeeb W, Kraivaphan P, Punyasingh J, et al. Oral lichen sclerosus et atrophicus. A case report. Oral Surg Oral Med Oral Pathol Oral Radiol Endod 1999; 88: 702-706.

13 Jiménez Y, Bagán JV, Milián MA, et al. Lichen sclerosus et atrophicus manifesting with localized loss of periodontal attachment. Oral Dis 2002; 8: 310-313.

14 Kaur S, Thami GP, Kanwar AJ, et al. Linear oro-facial lichen sclerosus. Clin Exp Dermatol 2002; 27: 467-470.

15 Chaudhry SI, Morgan PR, Neill SM. An unusual tongue. Clin Exp Dermatol 2006; 31: 831-832.

16 Jiménez Y, Gavaldá C, Carbonell E, et al. Lichen sclerosus of the oral mucosa: a case report. Med Oral Patol Oral Cir Bucal 2008; 13: E403-E406.

17 Azevedo RS, Romañach MJ, de Almeida OP, et al. Lichen sclerosus of the oral mucosa: clinicopathological features of six cases. Int J Oral Maxillofac Surg 2009; 38: 855-860.

18 Ravits HG, Welsh AL. Lichen Sclerosus et Atrophicus of the Mouth. Arch Dermatol 1957; 76: 56-58.

19 Brown AR, Dunlap CL, Bussard DA, et al. Lichen sclerosus et atrophicus of the oral cavity. Oral Surg Oral Med Oral Pathol Oral Radiol Endod 1997; 84: 165-170.

20 Schulten EAJM, Starink TM, van der Waal I. Lichen sclerosus et atrophicus involving the oral mucosa: report of two cases. $J$ Oral Pathol Med 1993; 22: 374-377.

$21 \mathrm{Liu}$ Y, Hua H, Gao Y. Oral lichen sclerosus et atrophicus literature review and two clinical cases. Chin J Dent Res 2013; 16: $157-160$.

22 Kelly SC, Helm KF, Zaenglein AL. Lichen sclerosus of the lip. Pediatr Dermatol 2006; 23: 500-502.

23 de Aquino Xavier FC, Prates AA, Gurgel CA, et al. Oral lichen sclerosus expressing extracellular matrix proteins and IgG4positive plasma cells. Dermatol Online J 2014; 20.

24 Marangon Júnior $\mathrm{H}$, Souza PEA, Soares RV, et al. Oral lichen sclerosus: a rare case report and review of the literature. Head Neck Pathol 2017; 11: 212-218. 
25 Gupta PC, Sinor PN, Bhonsle RB, et al. Oral submucous fibrosis in India: a new epidemic? Natl Med J India 1998; 11: 113-116.

26 Leitenberger JJ, Cayce RL, Haley RW, et al. Distinct autoimmune syndromes in morphea: a review of 245 adult and pediatric cases. Arch Dermatol 2009; 145: 545-550.

27 Gambichler T, Belz D, Terras S, et al. Humoral and cellmediated autoimmunity in lichen sclerosus. Br J Dermatol 2013; 169: 183-184.

28 Kreuter A, Kryvosheyeva Y, Teras S, et al. Association of autoimmune diseases with lichen sclerosus in 532 male and female patients. Acta Derm Venereol 2013; 93: 238-241.

29 Thomas R, Ridley CM, Mcgibbon DH, et al. Lichen sclerosus et atrophicus and autoimmunity - a study of 350 women. $\mathrm{Br} \mathrm{J}$ Dermatol 1988; 118: 41-46.

30 Cooper SM, Ali I, Baldo M, et al. The association of lichen sclerosus and erosive lichen planus of the vulva with autoimmune disease: a case-control study. Arch Dermatol 2008; 144: $1432-1435$.

31 Lipscombe TK, Wayte J, Wojnarowska F, et al. A study of clinical and aetiological factors and possible associations of lichen sclerosus in males. Australas J Dermatol 1997; 38: 132-136.

32 Azurdia RM, Luzzi GA, Byren I, et al. Lichen sclerosus in adult men: a study of HLA associations and susceptibility to autoimmune disease. Br J Dermatol 1999; 140: 79-83.

33 Oyama N, Chan I, Neill SM, et al. Autoantibodies to extracellular matrix protein 1 in lichen sclerosus. Lancet 2003; 362: 118-123.

34 Edmonds EVJ, Oyama N, Chan I, et al. Extracellular matrix protein 1 autoantibodies in male genital lichen sclerosus. $\mathrm{Br} J$ Dermatol 2011; 165: 218-219.

35 Sherman V, McPherson T, Baldo M, et al. The high rate of familial lichen sclerosus suggests a genetic contribution: an observational cohort study. J Eur Acad Dermatol Venereol 2010; 24: 1031-1034

36 Powell J, Wojnarowska F, Winsey S, et al. Lichen sclerosus premenarche: autoimmunity and immunogenetics. $\mathrm{Br} \mathrm{J}$ Dermatol 2000; 142: 481-484.

37 Sentürk N, Aydin F, Birinci A, et al. Coexistence of HLA-B*08 and HLA-B*18 in four siblings with lichen sclerosus. Dermatology 2004; 208: 64-66.

38 Aslanian FMNP, Marques MTQ, Matos HJ, et al. HLA markers in familial lichen sclerosus. J Dtsch Dermatol Ges 2006; 4: 842 847.

39 Gao XH, Barnardo MC, Winsey S, et al. The association between HLA DR, DQ antigens, and vulval lichen sclerosus in the UK: HLA DRB $1 * 12$ and its associated DRB $1 * 12 /$ DQB1*0301/04/09/010 haplotype confers susceptibility to vulval lichen sclerosus, and HLA DRB $1 * 0301 / 04$ and its associated DRB1*0301/04/DQB1*0201/02/03 haplotype protects from vulval lichen sclerosus. J Invest Dermatol 2005; 125: 895-899.
40 Marren $\mathrm{P}$, Jell J, Charnock FM, et al. The association between lichen sclerosus and antigens of the HLA system. $\mathrm{Br} J$ Dermatol 1995; 132: 197-203.

$41 \mathrm{Kim} \mathrm{CY,} \mathrm{Kim} \mathrm{JG,} \mathrm{Oh} \mathrm{CW.} \mathrm{Treatment} \mathrm{of} \mathrm{oral} \mathrm{lichen} \mathrm{sclerosus}$ with 1\% pimecrolimus cream. Ann Dermatol 2010; 22: 326-329.

42 Dickie RJ, Horne CHW, Sutherland HW, et al. Direct evidence of localised immunological damage in vulvar lichen sclerosus et atrophicus. J Clin Pathol 1982; 35: 1395-1397.

43 Baldo M, Bailey A, Bhogal B, et al. T cells reactive with the NC16A domain of BP180 are present in vulval lichen sclerosus and lichen planus. J Eur Acad Dermatol Venereol 2010; 24: 186-190.

44 Howard A, Dean D, Cooper S, et al. Circulating basement membrane zone antibodies are found in lichen sclerosus of the vulva. Australas J Dermatol 2004; 45: 12-15.

45 Rajlawat BP, Triantafyllou A, Field EA, et al. Lichen sclerosus of the lip and buccal mucosa. Clin Exp Dermatol 2004; 29: 684-685.

46 Sauder MB, Linzon-Smith J, Beecker J. Extragenital bullous lichen sclerosus. J Am Acad Dermatol 2014; 71: 981-984.

47 Tupsakhare S, Patil K, Patil A, et al. Lichen sclerosus of soft palate: a rare case report. Indian J Pathol Microbiol 2016; 59: 216-219.

48 Kirtschig G, Becker K, Günthert A, et al. Evidence-based (S3) Guideline on (anogenital) Lichen sclerosus. J Eur Acad Dermatol Venereol 2015; 29: e1-e43.

49 van der Nieuwenhof HP, Bulten J, Hollema H, et al. Differentiated vulvar intraepithelial neoplasia is often found in lesions previously diagnosed as lichen sclerosus, which have progressed to vulvar squamous cell carcinoma. Mod Pathol 2011; 24: 297-305.

50 Sherlin HJ, Ramalingam K, Natesan A, et al. Lichen sclerosus of the oral cavity. Case report and review of literature. $J$ Dermatol Case Rep 2010; 4: 38-43.

51 Bevans SL, Keeley JM, Sami N. Oral lichen sclerosus-a review of clinical presentation, treatment, and clinical outcomes. Oral Surg Oral Med Oral Pathol Oral Radiol 2017; 124: e243-e248.

\section{Answers to questions}

$1: d, 2: b, 3: b, 4: a, b, c, 5: c, d, 6: a, 7: b, 8: d, 9: b, c, 10: b$

\section{Supporting Information}

Additional Supporting Information may be found in the online version of this article:

Table S1. Demographic and clinical characteristics of OLS patients.

Table S2. Histopathological features of OLS lesions. 